\title{
A CASE REPORT OF ADULT ACNE
}

\author{
Department of Dermatology, \\ Venereology and Allergology, \\ Faculty of Medicine, \\ Medical University - Pleven, \\ Bulgaria \\ ${ }^{1}$ Department of Obstetrics and \\ Gynecology, \\ Faculty of Medicine, \\ Medical University - Pleven, \\ Bulgaria \\ ${ }^{2}$ Medical Diagnostic Clinical \\ Laboratory, \\ University Hospital "Dr. G. Stranski" \\ Pleven, \\ Bulgaria
}

\section{Ivelina A. Yordanova,
Desislava D. Tsvetanova, \\ Ivelina A. Yordanova,
Desislava D. Tsvetanova, Diana D. Strateva', Pavlina D. Yordanova-Laleva ${ }^{2}$, Dimitar K. Gospodinov}

\author{
Corresponding Author: \\ Ivelina A. Yordanova \\ Department of Dermatology, Venereology \\ and Allergology, \\ Faculty of Medicine, \\ Medical University - Pleven \\ 1, St. Kliment Ohridski Str. \\ Pleven, 5800 \\ Bulgaria \\ e-mail: ivelina_yordanova@abv.bg
}

Received: July 27, 2016

Revision received: October 31, 2016

Accepted: December 20, 2016

\section{Summary}

Acne vulgaris is among the commonest inflammatory skin diseases affecting pilosebaceous units. It occurs mainly in puberty and affects adolescents at the age of 1419 years both females and males, in relation to sebum production of hair follicles under the action of sex hormones. Clinically the disease is presented with comedones, papules, pustules, nodules and scars in some cases. Seborrheic areas face, chest and back are affected. Recent epidemiologic studies have shown significant number of female patients aged over 25 years with acne and the term Adult onset acne (AOA) was established. The latter is manifested clinically in the lower third of the face. Primary etiological cause of AOA is a hormonal imbalance, mainly hyperandrogenemia. A case of a 25year-old woman with adult acne and elevated levels of testosterone and prolactine is presented in this paper.

Key words: acne vulgaris, adult onset acne, hyperandrogenemia

\section{Introduction}

Acne vulgaris is among the commonest inflammatory skin diseases affecting pilosebaceous units. It occurs mainly in puberty and affects adolescents at the age of 14-19 years both females and males, in relation to sebum production of hair follicles under the action of sex hormones. Clinically the disease is presented with comedones, papules, pustules, nodules and scars in some cases. Seborrheic areas, face, chest and back are affected [1, 2]. Pathogenesis of the disease consists of microbial colonization of pilosebaceous units with Propionibacterium acnes, hyperkeratinization and obstruction of sebaceous follicles as a result of abnormal keratinization of the infundibullar epithelium and subsequent perifollicular inflammation. One of the most important pathogenetic factors is the androgenic stimulation of sebaceous glands [3]. Significant number of female patients aged over 25 years with acne has shown in the recent epidemiologic studies [4-6]. The term Adult onset acne was established by Kaur et al. in 2006 [4]. 


\section{Case Presentation}

We present a 25-year-old woman who has been suffering from acne vulgaris since she was fifteen. She complained from painful acneiform eruptions, affecting mainly the lower third of her face. From medical history there were no data about disturbances in her menstruation, she had regular cycles. The age of menarche was 11 years and the patient had no pregnancies. She had negative family history for acne vulgaris. The patient reported improvement of the cutaneous lesions from sun exposure in summer. One year ago she had visited another dermatologist and was treated with topical antibiotics and sunscreens without significant effect. At the time, a gynecological examination revealed hormonal abnormalities and a six-month therapy with Carbegoline $0.5 \mathrm{mg} /$ daily was given. Nevertheless, the disease was found resistant to the therapy.

Physical examination did not reveal any abnormalities. The body mass index (BMI) was 19.1. Dermatological examination showed pathological skin lesions affecting the lower third of the face, cheeks and chin, and the back. They were presented mainly by painful papules, some pustules and a few comedones (Figures 1, 2). According to Global Acne Grading System (GAGS) the global score was 22 which is a presentation for moderate acne. Laboratory examinations showed results from hematological, biochemistry and urine analysis within normal ranges. Hormonal results were without deviations except for elevated levels of total testosterone $(0.81 \mathrm{ng} / \mathrm{ml})$ and prolactin (47.16 ng/ml). Dehydroepiandrosterone Sulfate (DHEA-S) and luteinizing hormone (LH)/follicle-stimulating hormone (FSH) ratio were in normal ranges. Thyroid-stimulating hormone (TSH), Thyroglobulin antibodies (TAT), Thyroid microsomal antibodies (MAT), triiodothyronine (T3) and thyroxine (T4) were in normal ranges too. There were no abnormalities in the index of insulin resistance. A microbiological examination of pustules showed a sterile culture. After consultation with a gynecologist no data of polycystic ovary syndrome were found. On the basis of the data from the medical history, clinical and laboratory examinations, the patient was diagnosed with a moderate Adult acne. Topical treatment with Clindamycin phosphate $10 \mathrm{mg} / \mathrm{g}$ and Tretinoin $0.25 \mathrm{mg} / \mathrm{g}$ gel once a day was administered. Systemic therapy with Bromokriptin mesylate $2.5 \mathrm{mg}$ and Ethinyl estradiol 0.03 $\mathrm{mg} /$ Drosiprenon $3 \mathrm{mg}$ per day was recommended by the gynecologist.

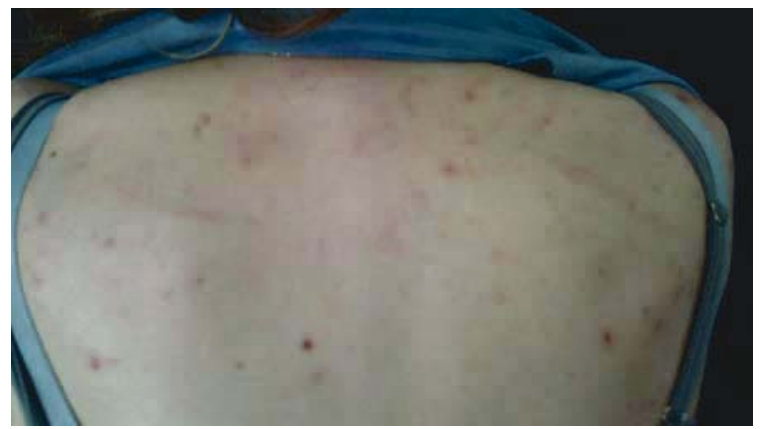

Figures 1, 2. Comedones and papules on the lower third on the face and back

As a result of the treatment at the end of the sixth month, slight improvement was observed. The eruptions on the face and back diminished in number and Global score was estimated 10 (Figures 3, 4). Despite the systemic treatment, hormonal results revealed still higher level of prolactine $(199.3 \mathrm{ng} / \mathrm{ml})$ and lower levels of estradiol $(10.0 \mathrm{pg} / \mathrm{ml})$ and progesterone $(1.28$ $\mathrm{ng} / \mathrm{ml})$ at the sixth month. The levels of total testosterone and DHEA-S were normal. The patient was directed to conduct magnetic resonance imaging, to exclude microadenoma of the pituitary gland.

\section{Discussion}

Adult onset acne (AOA) is defined as a chronic inflammatory disease of the pilosebaceous units, occurring at the age over 25 years [ $\underline{3}$ ]. It commonly affects females between the age of 25 and 50 years and is clinically presented with comedones, papules, pustules, cysts, and nodules on the lower part of the face, chin and jaw line [5, 


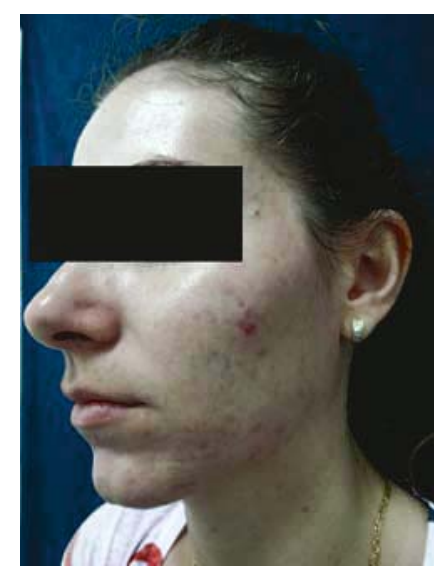

Figures 3, 4. Patient after six-month treatment

6]. Two different types of AOA are known: persistent acne and late-onset acne [4]. Persistent acne affects $82 \%$ of patients and is characterized by continuation of acne from adolescence to adult life [7]. Persistent acne is presented by painful inflammatory lesions, mainly papules and nodules involving lower third of face, jaw line and neck, as in the case presented. Late-onset acne appears for the first time after the age of 25 years and affects mainly the chin. Adult onset acne may be associated with a number of endocrine and non-endocrine diseases: polycystic ovary syndrome, insulin resistance and acanthosis nigricans syndrome, Cushing syndrome, metabolic syndrome, hyperandrogenemia, androgen-secreting tumors, Apert syndrome, pyogenic arthritis [8]. Commonly AOA is accompanied by hormonal imbalance. In women, the androgens deriving from the ovaries include androstenedione and testosterone. Dehydroepiandrosterone (DHEA), DHEA-S, androstenedione and testosterone are produced by the adrenal glands. Peripheral conversion of androstenedione and DHEA is also responsible for testosterone production in women. In female patients with AOA, signs and symptoms of hyperandrogenism (amenorrhea, oligomenorrhea, hirsutism, excessive discharge of sebum etc.) should be considered [9]. Fortyfive percent of adult women with AOA have hyperprolactinemia, which may be responsible for their excessive androgen signaling [5]. Skin androgen receptors are located in the sebaceous glands and in the outer root sheath of hair follicles. In the sebaceous glands, androgens undergo metabolization from DHEA to 5-alphadihydrotestosterone. This process includes many steps and is concerned with stimulating proliferation and activity of sebocytes [10].

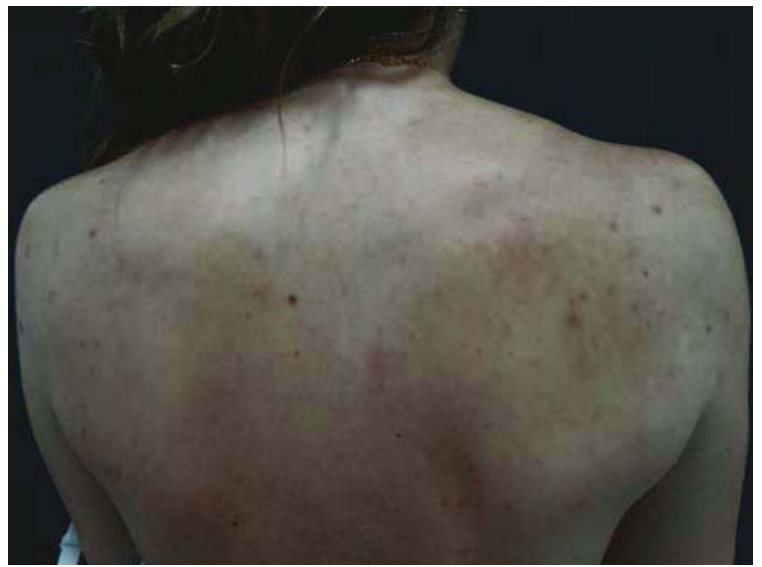

Sebum regulation is also related to a number of other hormones like estrogens, growth hormone, insulin and insulin-like growth factor-1, glucocorticoids, adrenocorticotropic hormone, and melanocortins [11]. Prolactin itself increases the level of 5-alpha-reductase in serum, which converts testosterone into dihydrotestosterone (DHT). On the other hand DHT increases sebocyte proliferation, sebum production and hyperkeratinisation [12]. Androgen levels often decrease when treatment for hyperprolactinemia is started.

Adult onset acne is mainly mild-to-moderate in severity, according to GAGS. This is a system introduced by Doshi et al. in 1997, for assessing the severity of acne, which divides the face, chest and back into six areas and assigns a factor to each area on the basis of size (Table 1). Each type of lesion (comedones, papules, pustules, nodules) is given a value $0-4$ depending on severity. The score for each area (local score) is calculated using the formula: Local score $=$ Factor $x$ Grade (0-4). The global score is the sum of local scores, and acne severity was graded using the global score. A score of $1-18$ is considered mild, 19-30 - moderate, 31-38 severe and more than 39 - very severe acne [13, 14].

When putting the diagnosis AOA, cosmetic acne, pomade acne, medication-induced acne, rosacea, perioral dermatitis and seborrheic dermatitis as differential diagnosis have to be in mind [15-17].

Most of the cases of AOA are resistant to the therapy, as in the case here presented [12]. Because hyperandrogenemia was evident in a majority of studies, these data suggest that androgen suppression may be useful in treating 
Table 1. Global Acne Grading System - division of the face, chest and back into six areas

\begin{tabular}{ll}
\hline Area & Factor \\
\hline Forehead & 2 \\
\hline Right cheek & 2 \\
\hline Left cheek & 2 \\
\hline Nose & 1 \\
\hline Chin & 1 \\
\hline Chest and upper back & 3 \\
\hline
\end{tabular}

acne [18]. Therapeutic approach with antiandrogens and other hormones (cyproteroneacetate, spironolactone, oral contraceptives, finasteride) are administered as a concomitant treatment for adult acne in woman. Patient selection and evaluation before treatment is administered as crucial [19]. Reducing sebum production is a major goal of hormonal treatment. Oral contraceptives may act through several mechanisms to reduce acne [20]. They stimulate synthesis of sex-hormone-binding globulin in the liver. This globulin binds circulating androgens, thus decreasing free testosterone and DHEA-S and probably contributes to lower sebum production. Oral contraceptives also inhibit 5-alfa-reductase, thus decreasing peripheral testosterone conversion and production of ovarian and adrenal androgens. These contraceptives have been proven to reduce lesion counts by $40-70 \%$, as demonstrated by results from a number of studies [4, 19]. Drospirenone (DRSP) has antimineralocorticoid and antiandrogenic effects and is particularly well tolerated, because it has no effect on fluid retention, compared to other oral contraceptives. Additionally, the relative safety effect of progestins has been demonstrated in large epidemiological studies [20].

\section{Conclusions}

We present a case-report of a 25-year-old female patient with moderate persistent AOA accompanied by hyperandrogenemia and hyperprolactinemia. After six-month systemic therapy with Bromokriptin mesylate, Ethinyl estradiol and Drosiprenon combined with retinoid and antibiotic topical therapy, a satisfactory result was reported. Regardless of the systemic therapy with antiandrogens, at the sixth month even higher level of prolactin has been found. Therefore the patient was directed to conduct magnetic resonance imaging, in order to exclude microadenoma of the pituitary gland.

\section{Acknowledgments}

This case report describes results from scientific research project № 8/2016, funded by Medical University - Pleven "Substantiation the relationship between acne vurgaris, polycystic ovary syndrome and disturbances in the function of the thyroid gland".

\section{References}

1. Cunliffe WJ, Gould DJ. Prevalence of facial acne vulgaris in late adolescence and in adults. Br Med J. 1979;1(6171):1109-10.

2. Ramos-e-Silva M, Ramos-e-Silva S, Carneiro S. Acne in women. Br J Dermatol. 2015;172(1):20-6.

3. Seirafi H, Farnaghi F, Vasheghani-Farahani A, Alirezaie NS, Esfahanian F, Firooz A, et al. Assessment of androgens in women with adultonset acne. Int J Dermatol. 2007;46(11):1188-91.

4. Kaur S, Verma P, Sangwan A, Dayal S, Jain VK. Etiopathogenesis and therapeutic approach to adult onset acne. Am J Clin Dermatol. 2006; 7(5):281-90.

5. Kamangar F, Shinkai K. Acne in the adult female patient: a practical approach. Int $\mathrm{J}$ Derm. 2012;51:1162-74.

6. Dréno B, Layton A, Zouboulis CC, LópezEstebaranz JL, Zalewska-Janowska A, Bagatin E, et al. Adult female acne: a new paradigm. J Eur Acad Dermatol Venereol. 2013;27(9):1063-70.

7. Williams C, Layton AM. Persistent acne in women: implications for the patient and for 
therapy. Am J Clin Dermatol. 2006;7(5):281-90.

8. Lolis MS, Bowe WP, Shalita AR. Acne and systemic disease. Med Clin North Am. 2009;93:1161-81.

9. Harper JC. Evaluating hyperandrogenism: a challenge in acne management. J Drugs Dermatol. 2008;7:527-30.

10. George R, Clarke S, Thiboutot D. Hormonal therapy for acne. Semin Cutan Med Surg. 2008;27:188-96.

11. Mualla P, Ekşioğlu M. Serum growth hormone and insulin-like growth factor-1 levels in women with postadolescent acne. Turkderm. 2010;44:69-72.

12. Rivera R, Guerra A. Management of acne in women over 25 years of age. Actas Dermosifiliogr. 2009;100:33-7.

13. Adityan B, Kumari R, Thappa DM. Scoring systems in acne vulgaris. Indian $\mathrm{J}$ Dermatol Venereol Leprol. 2009;75:323-6.

14. Hacivelioglu S, Gungor AN, Gencer M. Acne severity and the Global Acne Grading System in polycystic ovary syndrome. Int J Gynaecol Obstet. 2013;123(1):33-6.
15. Lehmann PM. Rosacea - epidemiology, pathogenesis, clinical presentation, and treatment. Dtsch Arztebl. 2007;104:1741-6.

16. Ljubojević S1, Lipozencić J, Turcić P. Perioral dermatitis. Acta Dermatovenerol Croat. 2008;16:96-100.

17. Schwartz RA, Janusz CA, Janniger CK. Seborrheic dermatitis: an overview. Am Fam Physician. 2006;74:125-30.

18. Haider A, Shaw JC. Treatment of acne vulgaris. JAMA. 2004;292:726-35.

19. Steinkellner A, Chen W, Denison SE. Adherence to oral contraception in women on Category $\mathrm{X}$ medications. Am J Med. 2010;123:929-34.

20. Tan JK, Ediriweera C. Efficacy and safety of combined ethinyl estradiol/drospirenone oral contraceptives in the treatment of acne. Int $\mathrm{J}$ Womens Health. 2010;9:213-21. 